\title{
A case of Merkel cell carcinoma of the head and neck
}

\author{
Sangwoo Suk, \\ Hyun Woo Shin, \\ Kun Chul Yoon \\ Department of Plastic and \\ Reconstructive Surgery, Kangbuk \\ Samsung Hospital, Sungkyunkwan \\ University School of Medicine, Seoul, \\ Korea
}

Merkel cell carcinoma (MCC) is a relatively rare and aggressive cutaneous neuroendocrine malignancy. It is characterized by high rates of recurrence and metastasis, both to regional lymph nodes and to distant locations. Its characteristic clinical manifestation is a single, painless, hard, erythematous nodule on a sun-exposed area, particularly in older men. Surgical management of both the primary site and the sentinel lymph node is the standard of care. In this article, we describe the diagnosis and treatment of a case of MCC in the left cheek.

Keywords: Carcinoma / Merkel cells / Skin neoplasm

\section{INTRODUCTION}

Merkel cell carcinoma (MCC), first described in 1972 by Toker [1], is a relatively rare and aggressive cutaneous neuroendocrine malignancy. It is characterized by high rates of recurrence and metastasis, both to regional lymph nodes and to distant locations. MCC most commonly presents as single, painless, hard, erythematous nodules on sun-exposed areas on the head or neck of older men. It is easily confused with other cancers of the skin, such as metastases of squamous cell carcinoma, melanoma, and other tumors. The current National Comprehensive Cancer Network (NCCN) guidelines recommend wide local excision of the primary site with 1-2 cm margins and sentinel lymph node (SLN) dissection. However, controversy exists regarding the outcomes of MCC treatment, and a multidisciplinary approach is needed for effective disease management.

\section{CASE REPORT}

A 77-year-old woman visited a tertiary hospital complaining of

\author{
Correspondence: Hyun Woo Shin \\ Department of Plastic and Reconstructive Surgery, Kangbuk Samsung Hospital, \\ Sungkyunkwan University School of Medicine, 29 Saemunan-ro, Jongno-gu, Seoul \\ 03181, Korea \\ E-mail: mdshin7@naver.com \\ Received October 1, 2019 / Revised December 6, 2019 / Accepted December 16, 2019
}

a single, painless, firm, erythematous, and rapidly-growing nodule on her left cheek area (Fig. 1). Due to her age and clinical characteristics, an excisional biopsy was performed at the dermatology department, and MCC was diagnosed by histopathology. Her medical history included surgery for mucoepidermoid carcinoma of the right salivary gland 20 years previously.

The patient was referred to the plastic surgery department of our hospital for further evaluation and treatment. Computed tomography (CT) and positron emission tomography (PET) scans were performed preoperatively to evaluate the extent of the primary tumor and to detect metastases. The CT scan revealed a subcutaneous enhancing nodular lesion measuring approximately $7 \times 5 \mathrm{~mm}$ in the left cheek area, and there was no evidence of a metastatic lesion in the neck area (Fig. 2A) However, the PET scan showed a hypermetabolic lymph node in the left side of the neck, at level II (Fig. 2B).

The treatment plan was designed using a multidisciplinary approach involving collaboration of head and neck surgery, hematology/oncology, radiation oncology, radiology, and nuclear medicine teams. Wide excision with regional neck lymph node dissection was planned.

First, the head and neck surgery team performed regional neck lymph node dissection. The wide local excision was completed by the plastic surgery team. The excision extended down to the level of the underlying muscular fascia to ensure a safety 


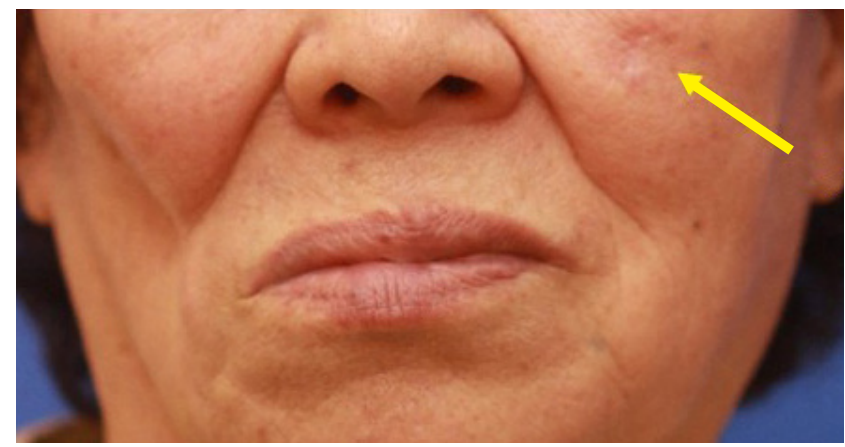

Fig. 1. The patient presented with a single, painless, firm, erythematous, and rapidly growing nodule. Yellow arrow indicated lesion on left cheek area.
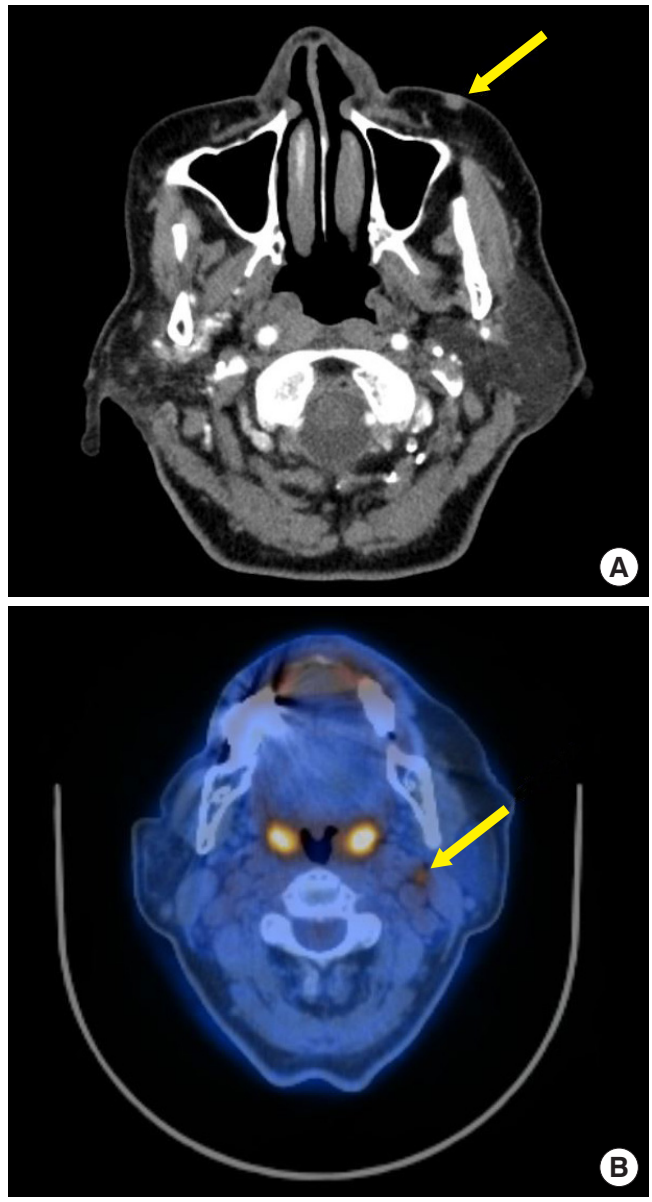

Fig. 2. A computed tomography scan and positron emission tomography scan were done. (A) Yellow arrow revealed a subcutaneous enhancing nodular lesion measuring approximately $7 \times 5 \mathrm{~mm}$ in the left check area. (B) Yellow arrow showed a hypermetabolic lymph node in the left neck, at level II.

margin of $2 \mathrm{~cm}$. Frozen section biopsies revealed no evidence of residual tumor cells. After tumor resection, a defect measuring approximately $5 \times 4 \mathrm{~cm}$ remained on the left anterior cheek area. We decided to use a radial forearm free flap to reconstruct
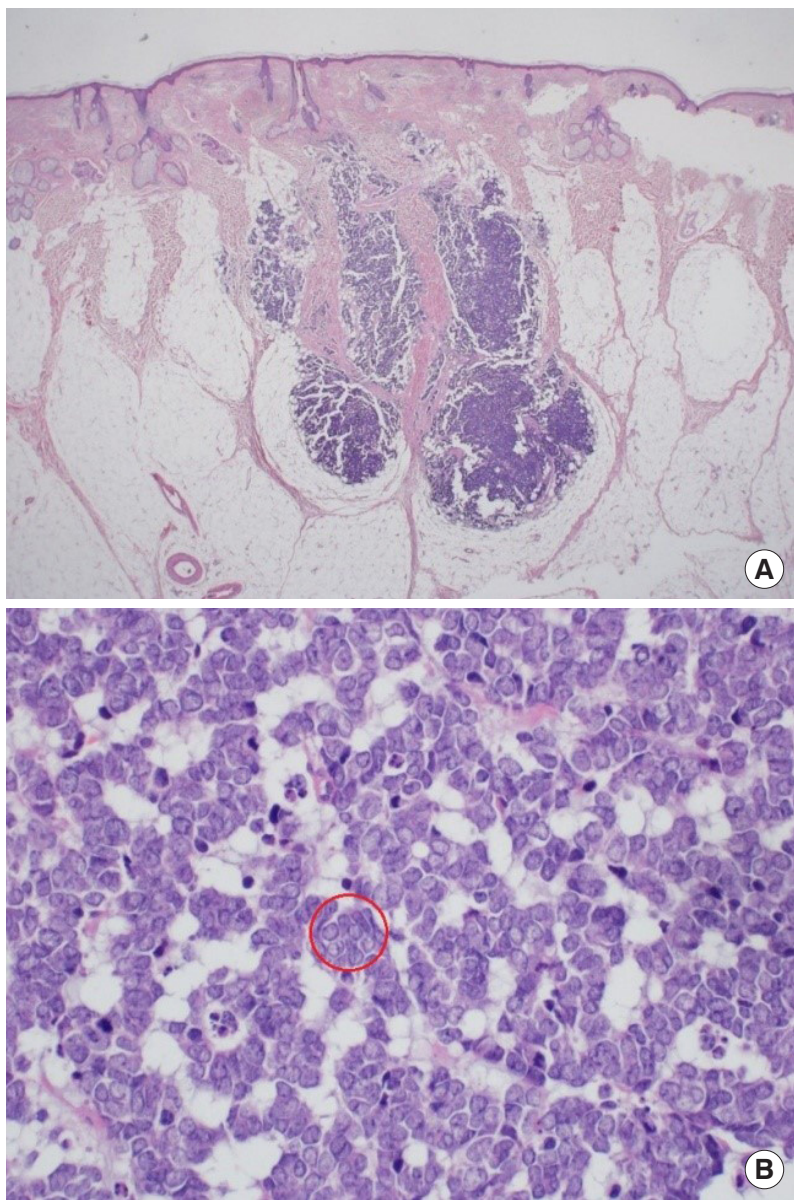

Fig. 3. A histopathologic examination confirmed Merkel cell carcinoma. Red circle indicated multifocal small tumor nests of monotonous round blue cells. (A) $\mathrm{H} \& \mathrm{E}, \times 12.5$; (B) $\mathrm{H} \& \mathrm{E}, \times 400$.

the defect area. The radial artery was anastomosed end-to-end with the superior thyroid artery, and the superior thyroid vein was anastomosed end-to-end with the vena comitans. The flap survived without any complications. The patient was discharged after 1 month.

A histopathologic examination revealed small multifocal tumor nests containing monotonous round blue cells in the subcutaneous layer. This highly infiltrative tumor was composed of small clusters of atypical plump spindle cells containing pleomorphic epithelioid and giant multinucleated cells in varying proportions, numerous mitotic figures, and infiltrative tumor margins (Fig. 3). The pathology findings were the same as reported in the first biopsy. Immunohistochemical staining demonstrated that the tumor cells were positive for cytokeratin-20 (CK-20), with a characteristic paranuclear dot pattern (Fig. 4). The resection margins were clear, with the closest safety margin and deepest margin measuring $1.5 \mathrm{~cm}$ and $0.1 \mathrm{~cm}$, respectively. Regional lymph node dissection did not reveal any metastases.

One month after excision, radiation therapy (RT) was admin- 


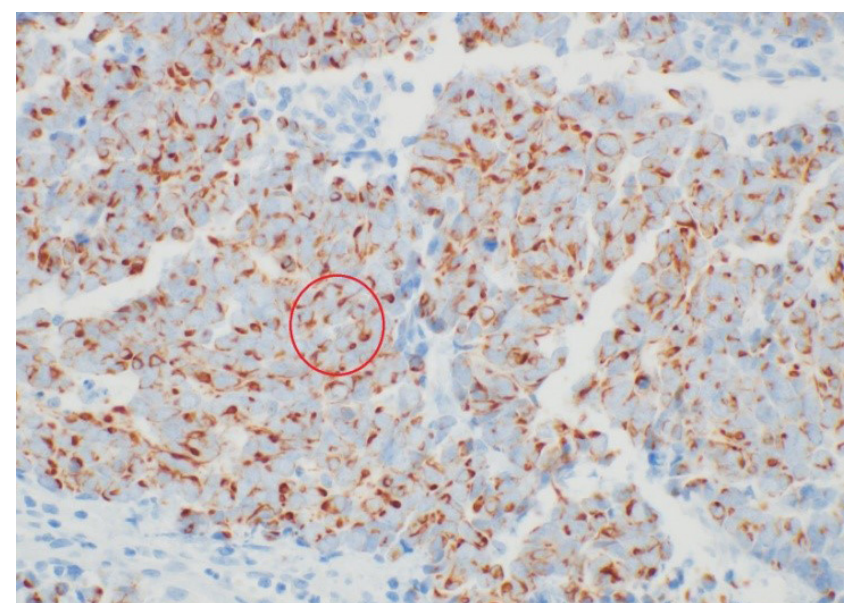

Fig. 4. A cytokeratin-20 stain was done and red circle revealed dotlike positivity in the tumor cells.

istered at the primary site, with a dose of $200 \mathrm{cGy} /$ fraction and a total dose of 5,400 cGy. RT was completed without any unusual adverse reactions.

At a 12-month postoperative follow-up examination, there was no evidence of systemic relapse on a CT scan. An examination of the surgical scar and neck lymph nodes did not reveal any local recurrence (Fig. 5).

\section{DISCUSSION}

Due to the rarity of MCC and its poorly understood etiology, clinical investigations in cases of MCC are often delayed [2]. However, the incidence of MCC has been steadily increasing in recent years. The overall age-adjusted worldwide incidence of MCC based on population studies from the Surveillance, Epidemiology, and End Results program in the United States, ranges from 0.10 to 1.6 cases per 100,000 people per year [3,4]. Epidemiologists have attributed this rise, in part, to improvements in diagnostic techniques, most notably CK-20 immunohistochemical staining [5]. CK-20 is low-molecular-weight keratin that is normally expressed in gastrointestinal epithelium, but also in some gastrointestinal and transitional cell carcinomas. CK-20 was the first immunostaining technique used to diagnose MCC reliably [6]. MCC tumors are usually CK-20-positive, making this feature useful for distinguishing MCC from other skin cancers. In our case, the tumor cells showed positivity for CK-20, although it is important to note that CK-20 expression has been reported to be negative in $5 \%-10 \%$ of MCC cases.

Surgical management of both the primary site and SLN is the standard of treatment for MCC. The current NCCN guidelines recommend wide local excision of the primary site with 1 to 2

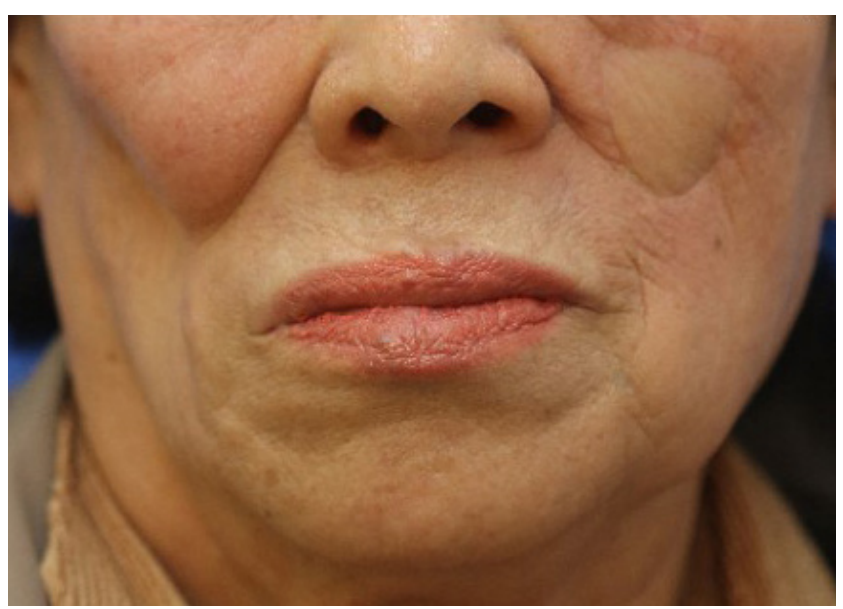

Fig. 5. At a 9-month postoperative follow-up visit, the left cheek flap had completely healed, with minimal scar contracture.

$\mathrm{cm}$ margins to the investing fascia of muscle when clinically feasible. Additionally, the NCCN recommends lymph node dissection for clinically evident lymphadenopathy. However, the management of positive SLNs remains controversial. Lemos et al. [7] conducted a retrospective analysisof5,823 cases and reported that $27 \%$ of cases presented with nodal disease. Poulsen et al. [8] showed that PET scans had sufficiently high sensitivity and specificity for MCC staging. The use of pretreatment PET scans for staging influenced treatment decisions by allowing upstaging of the disease in $25.9 \%$ of MCC patients. In our case, although we did not perform a preoperative SLN biopsy and no lymphadenopathy was detected, we carried out a wide local excision with neck dissection given the hypermetabolic lymph node detected on the preoperative PET scan and because of the high incidence of MCC recurrence.

Numerous retrospective studies and meta-analyses have attempted to determine whether postoperative RT improves outcomes in patients with MCC. Unfortunately, the results for both risks of recurrence/progression and survival are inconsistent. Therefore, it is unclear whether postoperative RT provides clinical benefits for node-negative patients. According to the NCCN guidelines, patients treated by wide excision with a negative SLN biopsy may undergo postoperative RT of the primary site or observation may be considered when the primary tumor is less than $1 \mathrm{~cm}$. Adjuvant chemotherapy is not recommended for local disease. In our case, we considered observation because of the small tumor size. However, we administered lowdose RT to reduce the risk of local recurrence.

Several large studies have documented the development of recurrence in approximately $25 \%-50 \%$ of all cases of MCC $[9,10]$. Therefore, close clinical follow-up of patients with MCC is important. The physical examination should include a complete 
skin and lymph node examination every 3 to 6 months for the first 3 years, then every 6 to 12 months thereafter. Imaging studies should be performed as clinically indicated, such as upon the development of new symptoms or lymphadenopathy. We conducted follow-up examinations every 3 months. At a 12-month postoperative follow-up examination, a CT scan was done and there was no evidence of recurrence.

We report a case of an MCC lesion in the head and neck. Due to the low incidence of MCC, it can be easily misdiagnosed as a benign mass on a clinical examination. However, the frequency of MCC has been increasing for several decades, and plastic surgeons should, therefore, be aware of this condition and be familiar with how to manage it appropriately. In addition, considering the high rate of recurrence ( $4 \%$ local and 6\% regional), surgeons should ensure that MCC patients receive regular follow-up visits after surgical treatment. Through this case report, we aim to educate the medical community on MCC, warn clinicians of the possibility of misdiagnosis, and provide information on the appropriate treatment.

\section{NOTES}

\section{Conflict of interest}

No potential conflict of interest relevant to this article was reported.

\section{Ethical approval}

The study was approved by the Institutional Review Board of Kangbuk Samsung Hospital (IRB No. KBSMC 2019-09-025) and performed in accordance with the principles of the Declaration of Helsinki. Written informed consent was obtained.

\section{Patient consent}

The patient provided written informed consent for the publication and the use of her images.

\section{ORCID}

$\begin{array}{ll}\text { Sangwoo Suk } & \text { https://orcid.org/0000-0002-7647-841X } \\ \text { Hyun Woo Shin } & \text { https://orcid.org/0000-0003-4396-3395 }\end{array}$
Kun Chul Yoon https://orcid.org/0000-0001-9167-6716

\section{REFERENCES}

1. Toker C. Trabecular carcinoma of the skin. Arch Dermatol 1972;105:107-10.

2. Lemos B, Nghiem P. Merkel cell carcinoma: more deaths but still no pathway to blame. J Invest Dermatol 2007;127:2100-3.

3. Agelli M, Clegg LX, Becker JC, Rollison DE. The etiology and epidemiology of Merkel cell carcinoma. Curr Probl Cancer 2010;34:14-37.

4. Fitzgerald TL, Dennis S, Kachare SD, Vohra NA, Wong JH, Zervos EE. Dramatic increase in the incidence and mortality from Merkel cell carcinoma in the United States. Am Surg 2015; 81:802-6.

5. Agelli M, Clegg LX. Epidemiology of primary Merkel cell carcinoma in the United States. J Am Acad Dermatol 2003;49:83241.

6. Moll R, Lowe A, Laufer J, Franke WW. Cytokeratin 20 in human carcinomas: a new histodiagnostic marker detected by monoclonal antibodies. Am J Pathol 1992;140:427-47.

7. Lemos BD, Storer BE, Iyer JG, Phillips JL, Bichakjian CK, Fang LC, et al. Pathologic nodal evaluation improves prognostic accuracy in Merkel cell carcinoma: analysis of 5823 cases as the basis of the first consensus staging system. J Am Acad Dermatol 2010;63:751-61.

8. Poulsen M, Macfarlane D, Veness M, Estall V, Hruby G, Kumar M, et al. Prospective analysis of the utility of 18-FDG PET in Merkel cell carcinoma of the skin: A Trans Tasman Radiation Oncology Group Study, TROG 09:03. J Med Imaging Radiat Oncol 2018;62:412-9.

9. Santamaria-Barria JA, Boland GM, Yeap BY, Nardi V, DiasSantagata D, Cusack JC Jr. Merkel cell carcinoma: 30-year experience from a single institution. Ann Surg Oncol 2013;20:136573.

10. Tarantola TI, Vallow LA, Halyard MY, Weenig RH, Warschaw KE, Grotz TE, et al. Prognostic factors in Merkel cell carcinoma: analysis of 240 cases. J Am Acad Dermatol 2013;68:425-32. 